that the summit of Mauna Kea has always had within the indigenous Hawaiian community. We are most fortunate to have the opportunity to conduct observations from this mountain. The data described in the paper are presented in the supplementary materials.
Tables S1 to S5

References (35-42)

1 June 2012; accepted 31 August 2012 $10.1126 /$ science. 1225506

\section{Imaging the Homogeneous Nucleation During the Melting of Superheated Colloidal Crystals}

\author{
Ziren Wang, ${ }^{1}$ Feng Wang, ${ }^{1}$ Yi Peng, ${ }^{1}$ Zhongyu Zheng, ${ }^{1,2}$ Yilong Han $^{1 *}$
}

The nucleation process is crucial to many phase transitions, but its kinetics are difficult to predict and measure. We superheated and melted the interior of thermal-sensitive colloidal crystals and investigated by means of video microscopy the homogeneous melting at single-particle resolution. The observed nucleation precursor was local particle-exchange loops surrounded by particles with large displacement amplitudes rather than any defects. The critical size, incubation time, and shape and size evolutions of the nucleus were measured. They deviate from the classical nucleation theory under strong superheating, mainly because of the coalescence of nuclei. The superheat limit agrees with the measured Born and Lindemann instabilities.

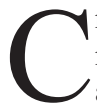
rystal melting is of considerable fundamental and practical importance to science and technology. Yet, our understanding of it is still rather incomplete. Thermodynamics reveals to us the equilibrium phases, but the kinetics of phase transitions have proved difficult to predict (1-3). Crystals melt heterogeneously from surfaces or grain boundaries once they are heated to the melting point $(3,4)$. By suppressing surface melting (5-8), a single crystal can be superheated to temperatures above its melting point. This metastable state will eventually melt from the interior without any preferential sites. In such homogeneous melting, small liquid nuclei form spontaneously by thermal fluctuations. A spherical nucleus has the free energy (9)

$$
\Delta G=4 \pi r^{2} \gamma-\frac{4}{3} \pi r^{3} n \Delta \mu+E_{\text {strain }}
$$

where $r$ is the radius, $\gamma$ is the surface tension, $n$ is the number density of the nucleus, $\Delta \mu(>0)$ is the chemical potential difference between the superheated crystal and liquid, $E_{\text {strain }}=\frac{4}{3} \pi r^{3} n \Delta \epsilon$ is the misfit strain energy in the crystal caused by the volume change of the nucleus, and $\Delta \epsilon$ is the mean strain energy per particle from continuum elasticity and is not expected to depend on $r . E_{\text {strain }}$ is zero when the parent phase is fluid but is finite when the parent phase is solid (10). To minimize $\Delta G$, small liquid nuclei tend to recrystallize rather than grow unless their size exceeds a critical value $r^{*}=2 \gamma /[(\Delta \mu-\Delta) n]$ corresponding to the barrier height of $\Delta G$.

${ }^{1}$ Department of Physics, Hong Kong University of Science and Technology, Clear Water Bay, Hong Kong SAR, China. Institute of Mechanics, Chinese Academy of Sciences, Beijing 100190, China.

${ }^{*}$ To whom correspondence should be addressed. E-mail: yilong@ust.hk
The small length and time scales of the nucleation process preclude observation at the singleparticle level in molecular systems. In contrast, micrometer-sized colloidal particles can serve as good model systems for phase transition studies because their thermal motions can be directly visualized and measured with video microscopy $(11,12)$. Crystallization (13), sublimation (14), and heterogeneous melting in polycrystals $(4,15)$ have been studied in colloids.

We used thermal-sensitive $N$-isopropylacrylamide fective diameter $\sigma$ linearly changes from $0.76 \mu \mathrm{m}$ at $26.4^{\circ} \mathrm{C}$ to $0.67 \mu \mathrm{m}$ at $30.6^{\circ} \mathrm{C}(10)$. The effective diameter is defined so that the melting volume fraction $\phi_{m}=54.5 \%$, which is the same as that of the hard spheres. By this definition, the measured freezing point $\phi_{f}=49 \%$, which is close to that of the hard spheres $\left(\phi_{f}^{H S}=49.4 \%\right)(10)$. At $49 \%<\phi<54.5 \%$, crystal and liquid coexist. The volume fraction in colloids plays a similar role to the temperature in molecular systems (16). Particles were loaded into an 18 by 3 by $0.1 \mathrm{~mm}^{3}$ glass channel and annealed to a single-facecentered cubic crystal or a polycrystal with only a few domains. The crystalline structure and the good refractive-index matching between particles and water enable us to see through all the 150 layers by means of bright-field microscopy (4). By changing the temperature, we can easily change the volume fraction to melt or recrystalize the sample repeatedly. However, because melting always starts heterogeneously from surfaces for a single crystal and from grain boundaries for a polycrystal (15), to create homogeneous melting the interior of a perfect crystalline domain was heated with a beam of light passing through an objective lens (Fig. 1A and movie S1) (10). The $4500-\mu \mathrm{m}^{2}$ heated area in the focal plane had a temperature $T_{\mathrm{amb}}+\delta T$, where the ambient tem(NIPA) microgel colloidal spheres (4), whose ef- perature $T_{\mathrm{amb}}$ was controlled by the temperature controller on the microscope with a resolution of $0.1^{\circ} \mathrm{C}$ (a volume fraction of $0.4 \%$ ), and $\delta T=2.0^{\circ} \mathrm{C}$ was the local optical heating effect, which was reached $2 \mathrm{~s}$ after the light was turned on (15). The crystal was superheated when $\Delta T \equiv T_{\mathrm{amb}}+\delta T-$ $T_{m} \equiv T-T_{m}>0$ - that is, $-\Delta \phi \equiv \phi_{\mathrm{amb}}-\delta \phi-\phi_{m} \equiv$ $-\left(\phi_{m}-\phi\right)<0$. Because the light was focused by the objective, the heating was the strongest in the focal plane and decayed to $0.9 \delta T$ at about \pm 30 layers in the $z$ direction (10) and was uniform in the center of the $x y$ plane (Fig. 1B, temperature profile). Nucleation started randomly in the $\pi(38 \mu \mathrm{m})^{2}$ by $( \pm 10 \mu \mathrm{m})$ region of interest, hence the melting was homogeneous. To capture the initial stage of the nucleation, we rapidly scanned \pm 10 layers along the $z$ direction within $2 \mathrm{~s}$ every $\sim 20 \mathrm{~s}$ and only recorded those nucleations that started near the focal plane. Nucleus volumes were measured from three-dimensional (3D) scans (movie S2) (10), while dynamics were measured by monitoring the largest 2D cross section of nucleus. The observation time scale ranged from 1 to $4000 \mathrm{~s}$, which enabled us to explore both the strong superheating where melting was catastrophic and the weak superheating where nucleation was rare. The particle motions were recorded with a charge-coupled device (CCD) camera at 15 frames per second. Particle positions were tracked from the image analysis (17) in the crystal phase but not in the liquid phase, owing to their blurry images. Experimental details are given in (10).

In the theory of defect-mediated melting, defects are generated from a perfect crystal as temperature increases. They diffuse, coalesce, and form nuclei above the melting point. There is controversy, however, over which type of defect is the homogeneous melting precursor. In contrast to the conventionally suggested dislocations $(18,19)$, interstitials, and vacancies $(20)$, recent simulations with different pair potentials showed that the precursors are collective loop motions when catastrophic melting at the superheating limit is avoided by using a slow heating rate $(21,22)$. In our colloidal samples, the heating rate was infinitely slow (the temperature was kept constant) so that the kinetics can be accurately measured. We also observed that the precursors were local particle exchanges instead of any obvious defects. As shown in Fig. 1, C and D, and movie S3 (10), particles marked by a white spot left their lattice sites, but the crystalline structure remained intact, which indicates that the particles swapped positions with their neighbors and formed closed loops in three dimensions. This is not surprising because it is the easiest way to move particles around in a perfect lattice. Moreover, we observed that local particle exchanges were surrounded by large-Lindemann-parameter 
Fig. 1. (A) The schematic of local optical heating. (B) The measured temperature profile in the focal plane under the light intensity used in most of the measurements, including Figs. 1, 2, and 3B. The contour spacing is $0.2^{\circ} \mathrm{C}$. The temperature difference in the $\pi$ (38 $\mu \mathrm{m})^{2}$ by $20 \mu \mathrm{m}$ region of interest is less than $0.2^{\circ} \mathrm{C}$. (C to I) A typical nucleation process at $\phi=$ $52.0 \%$ (i.e., $\Delta T=0.6^{\circ} \mathrm{C}$ ) whose size evolution is shown by curve 2 in Fig. $2 \mathrm{~A}$. The heating light was turned on at $t=0 \mathrm{~s}$. The colors represent different values of the Lindemann parameter. Liquid particles labeled in red are defined by the orientational order parameter $\psi \equiv$ $\left|\sum_{j=1}^{n n} e^{6 i \theta_{j}}\right| / n n<0.6$, where $\theta_{j}$ is the orientation of the bond between the particle and its neighbor $j$, and $n n$ is the number of nearest neighbors the particle has (15). (C) Two particles labeled in white spots left their lattice sites and swapped positions with their neighbors, but the lattice structure remained intact (movie S2) (10). (D) Three regions of particles in the midst of swapping positions surrounded by particles with large Lindemann parameter. (E) The regions with large Lindemann parameter particles coalesced
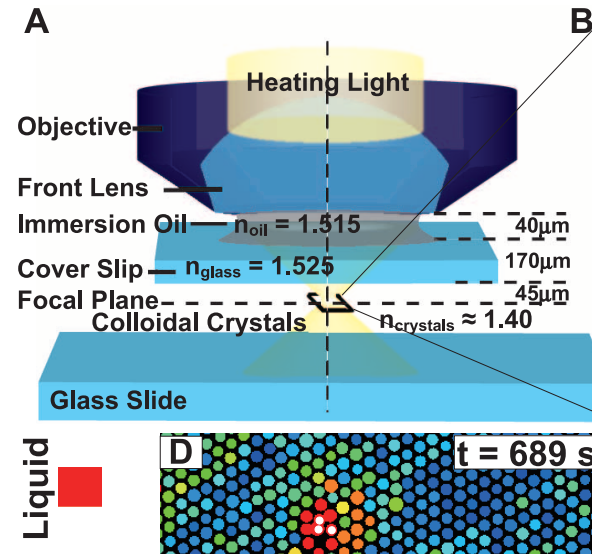
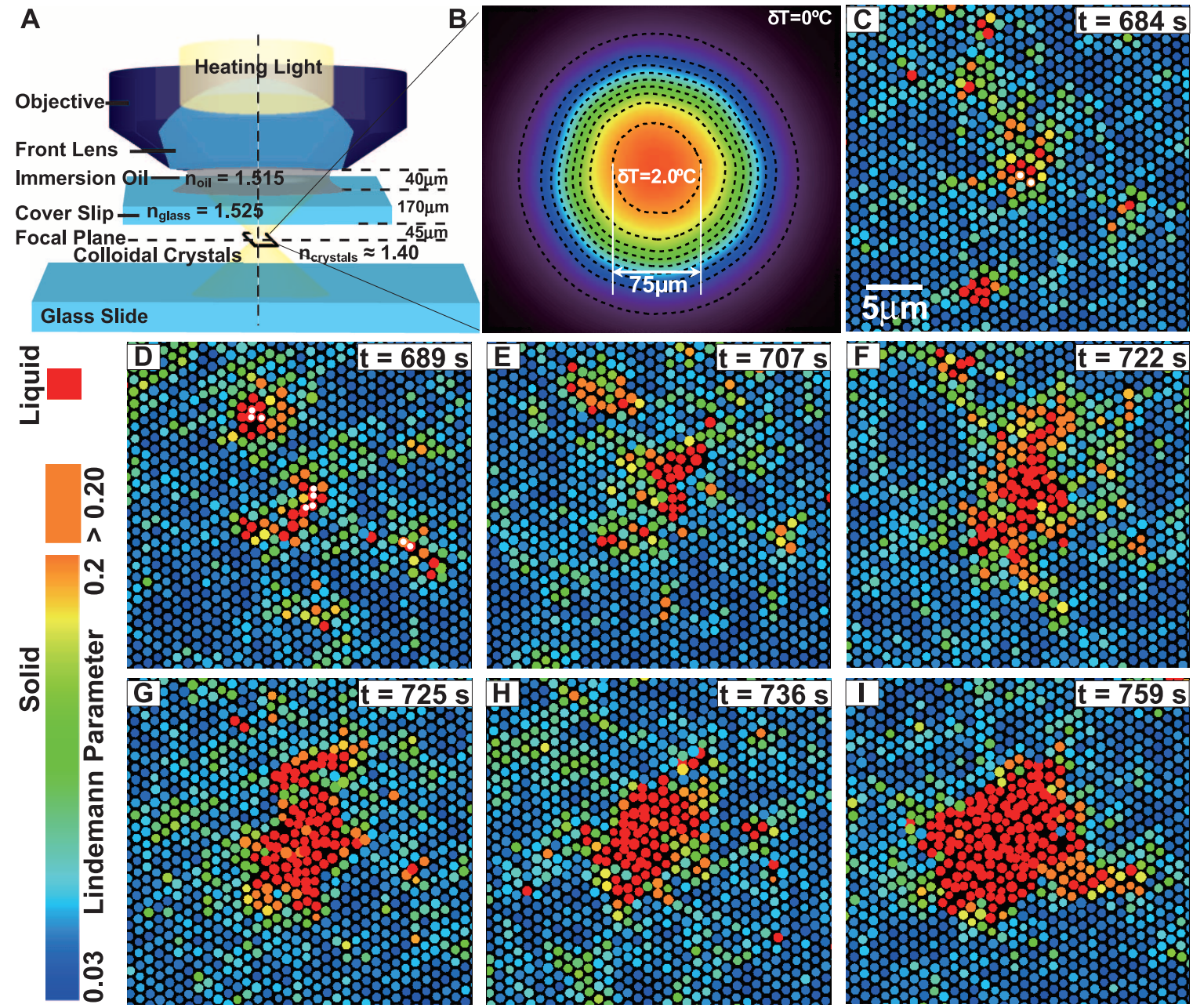

and formed a liquid nucleus. The nucleus reached the critical size in $(F)$ and grew into a noncircular postcritical nucleus in $(G)$, then became smaller and circular in (H) and rapidly expanded in (I). (large- $L$ ) particles. The colors in Fig. 1, C to I, represent the values of the Lindemann parameter $L$, which reflects the motion amplitude of the particle relative to its equilibrium position (15). The large- $L$ regions lasted for up to $1 \mathrm{~min}$ before they relaxed back to normal crystals or transformed into a liquid nucleus (Fig. 1E). Consequently, nucleation can be considered a two-step process: crystal $\rightarrow$ crystal with domains of large- $L$ particles $\rightarrow$ nucleation. This is in accordance with Ostwald's step rule (23), that intermediate metastable or unstable states commonly exist in real nucleation processes to lower the free-energy barrier between the child and parent phases.

On the basis of the nucleation behaviors, we divide the superheating range into three regimes: weak superheating $\left(\Delta T \lesssim 0.5^{\circ} \mathrm{C}\right.$, i.e., $\phi \gtrsim 52.5 \%$, no coalescence between nuclei), intermediate superheating $\left(0.5^{\circ} \mathrm{C} \lesssim \Delta T \lesssim 0.8^{\circ} \mathrm{C}\right)$, and strong superheating $\left(\Delta T \gtrsim 0.8^{\circ} \mathrm{C}\right.$ - that is, $\phi \lesssim 51.0 \%$, all nuclei irreversibly grow bigger). Figure $2 \mathrm{~A}$ shows some typical nucleus-size evolutions under the weak, intermediate, and strong superheating regimes. A newly formed nucleus can be large if it is formed in a large large- $L$ region (Fig. $2 \mathrm{~A}$, jumps in curves 1 and 4). Such nuclei are smaller than the critical size under weak superheating. Under strong superheating, however, the critical nucleus is small (Fig. 2B), so that all the nuclei irreversibly grow bigger, and there are no obvious subcritical nuclei. Under the intermediate superheating regime, both types of nucleation paths (with and without the subcritical stage) were observed.

Under weak superheating, the nucleation kinetics basically followed the classical nucleation theory: Subcritical nuclei formed and disappeared during the incubation period (which typically spanned $30 \mathrm{~min}$ to more than 1 hour) until one of them reached the critical size. The nucleus size usually changed slowly so that we had enough time to scan in the $z$ direction and measure the nucleus volume $V$ and the effective radius $r=$ $(3 V / 4 \pi)^{1 / 3}$ shown in Fig. 2B. The critical nucleus size $r^{*}$ was measured by adjusting $T_{\mathrm{amb}}$ so that a nucleus became as stable as possible with an equal probability of growing or shrinking (10). From Eq. $1, r^{*}(\Delta \phi) \sim(\Delta \phi-d \phi)^{-1}$ where $d \phi$ is the forbidden gap caused by the strain energy $(10,24)$. In this gap, the superheated crystal cannot transform into the lower-energy liquid phase because a kinetic path is not available $(24) . r^{*}(\Delta \phi)$ in Fig. $2 \mathrm{~B}$ can be accurately fitted for $d \phi<0.002$. This upper bound of the gap is smaller than our temperature resolution and comparable with our estimated $d \phi \approx$ 0.0046 for hard spheres (10). The small $d \phi$ is negligible, hence the critical nucleus $r^{*} \approx 2 \gamma /(n \Delta \mu)$, the chemical potential difference $\Delta \mu \approx L_{m} \Delta \phi /\left(\phi_{m}-\phi_{f}\right)$, where $L_{m}$ is the latent heat per particle usually taken as a constant (24) and the free energy barrier $\Delta G^{*} \approx$ $16 \pi \gamma^{3} /\left[3(n \Delta \mu)^{2}\right]$ under weak superheating (10). These relations yield $r^{*} \approx 2\left(\phi_{m}-\phi_{f}\right) \gamma /\left(n L_{m} \Delta \phi\right)$. Our measured $r^{*} \propto 1 / \Delta \phi$ in Fig. 2B, hence the surface tension $\gamma$ is approximately a constant (10). If we take hard sphere's $L_{m}=1.168 k_{\mathrm{B}} T$ (25), where $k_{\mathrm{B}}$ is the Boltzmann constant, then $\gamma=$ $0.84 k_{\mathrm{B}} T / \sigma^{2}$ and $\Delta \mu=23.8 k_{\mathrm{B}} T \Delta \phi$, which are comparable with those of hard sphere $(26,27)$. Comparing our fitted $r^{*}=0.156 \sigma\left(\phi_{m} / \Delta \phi\right)$ near $\phi_{m}$ from Fig. 2B and $r^{*}=0.156 \sigma\left(\phi_{f} / \Delta \phi\right)$ for the crystallization of hard spheres near $\phi_{f}(26)$, we found that melting and freezing have the same 


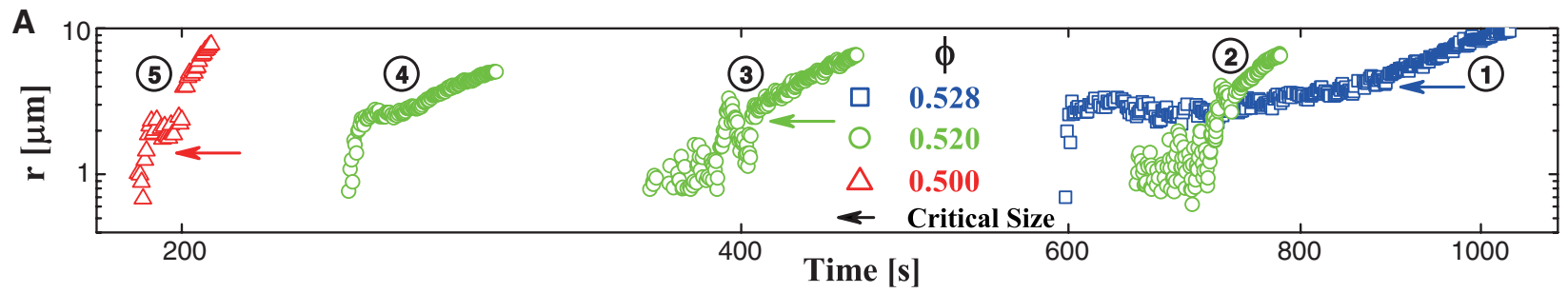

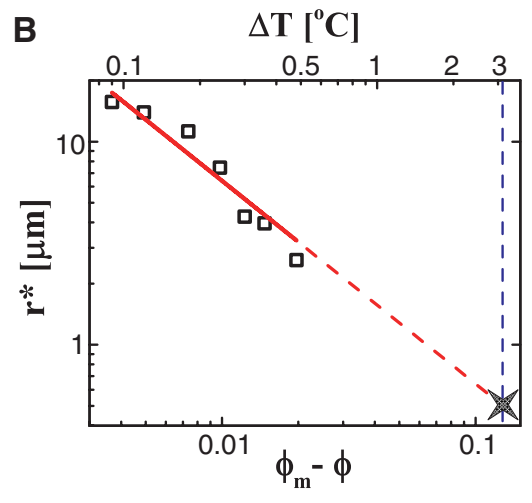

C

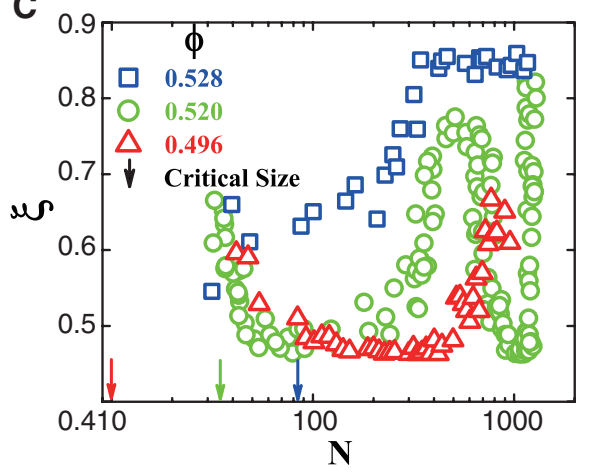

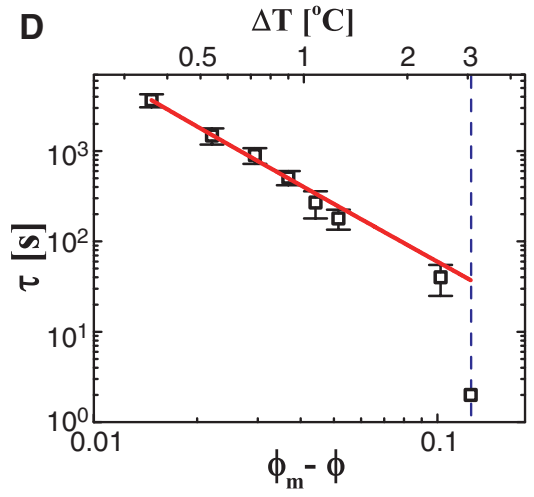

Fig. 2. (A) The evolution of the effective radius of nucleus at $\varphi=0.528(\Delta T=$ $0.4^{\circ} \mathrm{C}$, curve 1$), 0.520\left(\Delta T=0.6^{\circ} \mathrm{C}\right.$, curves 2,3 , and 4$)$, and $0.500\left(\Delta T=1.1^{\circ} \mathrm{C}\right.$, curve 5) (movie S4) (10). (B) The critical nucleus radius $r^{*}(\Delta \phi)$ (squares) is fitted by $(\Delta \phi)^{-1}$ (solid line) and extrapolated to the superheat limit. (C) The shape factor $\xi$ as a function of the particle number in the cross section of the nucleus for three nucleation processes at $\phi=0.528$ (blue squares), 0.520 (green circles), and 0.496 (red triangles). The two minima of the $\phi=0.520$ curve and the one minimum of the $\phi=0.496$ curve correspond to nuclei coalescence. (D) The incubation time $\tau$ (squares symbols) is fitted by $(\Delta \phi)^{-2}$ (solid line). The vertical dashed lines in (B) and (D) denote the superheat limit.
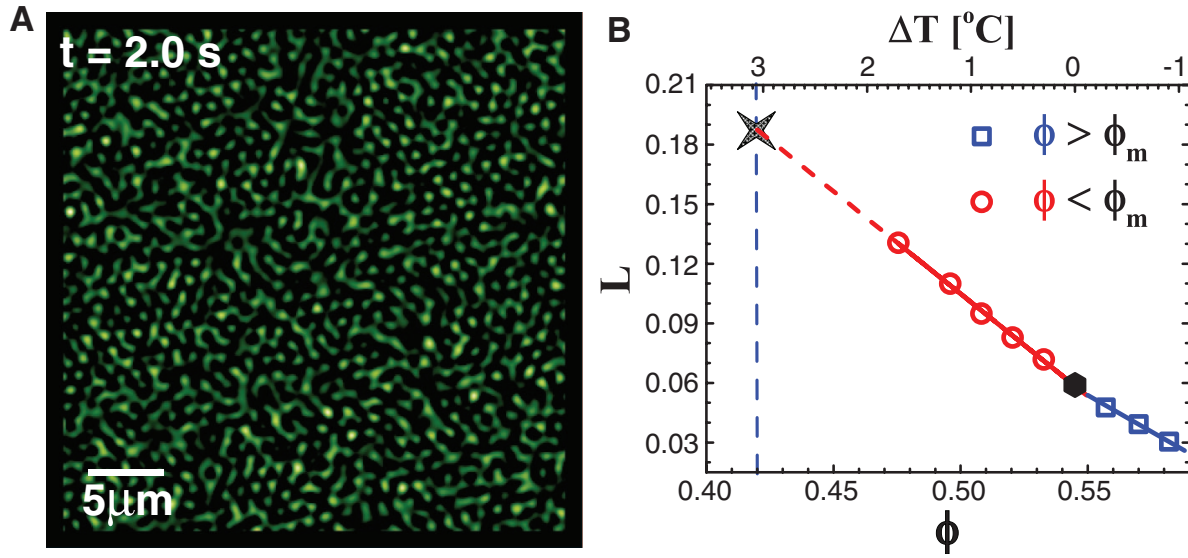

Fig. 3. (A) Melting started catastrophically from everywhere at $\phi=41 \%$. (B) The Lindemann parameter is extrapolated to the superheat limit $\phi_{s}=42 \%$.

prefactor in the colloidal system, although this result was only predicted in molecular systems (24).

Further complicating the nucleation kinetics, multiple large- $L$ regions or liquid nuclei can coalesce especially under strong superheating (movies S3 and S6) (10). After coalescence, the nuclei became more nonspherical but eventually relaxed into spheres after a few minutes (fig. S4) (10). We characterized the shape of the nucleus by measuring the 2D shape factor $\xi=4 \pi A / l^{2}$, where $A$ is the area and $l$ is the perimeter of the nucleus. $\xi=1$ for a circle and $<1$ for a noncircle. When a nucleus evolves without the influence of nearby nuclei or large- $L$ regions, it is more noncircular at small sizes because the shape is more vulnerable to thermal fluctuations (Fig. $2 \mathrm{C}$, the $\phi=0.528$ curve). Under strong superheating, nuclei often coalesce and become more noncircular in shape (Fig. $2 \mathrm{C}$, the $\phi=0.520$ and 0.496 curves). Noncircular nuclei tend to become smaller circular nuclei even when they are larger than the measured mean critical size (Figs. 1, G and $\mathrm{H}$, and 2A, the oscillations near the critical size in curves 2 and 3 ). Hence, noncircular nuclei appear to have larger critical sizes than circular ones (28), which is in line with the observation of the nucleus shrinking after coalescence in crystallization (29) because coalescence often makes a nucleus more noncircular.

We measured the incubation time $\tau$ as the time taken for the first post-critical nucleus to form (15). In our 90,000-particle region of interest, $\tau$ has large fluctuations (such as Fig. 2A, curves 2, 3 and 4). Hence, we averaged each $\tau$ in Fig. 2D over $\sim 15$ nucleation processes. The measured $\tau$ in Fig. 2D agree with the $\tau(\Delta \phi) \sim \Delta \phi^{-2}$ from the classical nucleation theory under weak superheating $(9)$ but become smaller under stronger superheating. We attribute this departure to the more frequent coalescence of nuclei under stronger superheating, which can speed up the incubation process (movie S5) (10).

The superheat limit of most molecular crystals is $\sim 1.2 T_{m}$ - crystals become unstable and catastrophically melt without incubation at $\sim 20 \%$ above their melting point $(6,7,30-32)$. However, the superheat limit of colloidal crystals is not known. Our colloidal crystals are metastable and melt via a nucleation mechanism, with a free energy barrier at $\phi=43 \%$, but become unstable and melt uniformly like a spinodal in $2 \mathrm{~s}$ at $\varphi=41 \%$ (Fig. 3A and movie S6) (10), hence the superheat limit is at $\phi_{s}=42 \pm 1 \%$. This is $23 \%$ lower than $\phi_{m}=54.5 \%$, analogous to the supercool limit of hard spheres at the glass transition point $\phi_{g}^{H S}=$ $58 \%$, which is $17 \%$ above $\phi_{f}^{H S}=49.4 \%$. In theory, the superheat limit may depend on a series of instability points of the crystalline state. These instability points are the kinetic instability (31), the Born criterion $(32,33)$, and the isochoric (meaning the liquid and the superheated solid have equal volume) (33), isentropic (equal entropy) (34), and the isenthalpic (equal enthalpy) (34) points. We found the superheat limit $42 \%$ is consistent with the measured Lindemann and Born instabilities $(2,31)$ but lower than the isochoric 
$\phi=\phi_{f}^{H S}=49.4 \%$, which is the isentropic and the isenthalpic $\phi=51 \%$ (26) of hard spheres. At $\phi_{s}=$ $42 \%$, the extrapolated Lindemann parameter $L_{\mathrm{S}}=0.187$ (Fig. 3B), which is close to $L=0.176$ at the crystal-liquid interfaces and $L_{\mathrm{s}} \cong 0.2$ for most molecular crystals (35). Moreover, the extrapolated $r_{\mathrm{s}}^{*}=0.59 a$ at $\phi_{\mathrm{s}}=42 \%$, where $a$ is the lattice constant (Fig. 2B). These results further confirm that $\phi_{\mathrm{s}}=42 \%$ where the crystal is about to break down from the single-particle scale.

This experimental system enables us to investigate complex dynamics during the $3 \mathrm{D}$ homogeneous melting, including the nucleation precursor and nucleus evolution. It also provides a platform to study single-defect effects on melting and homogeneous nucleation in solid-solid transitions, which are difficult to be measured in molecular crystals.

\section{References and Notes}

1. A. Laaksonen, V. Talanquer, D. Oxtoby, Annu. Rev. Phys. Chem. 46, 489 (1995).

2. R. W. Cahn, Nature 413, 582 (2001).

3. J. G. Dash, Rev. Mod. Phys. 71, 1737 (1999).

4. A. M. Alsayed, M. F. Islam, ]. Zhang, P. ]. Collings, A. G. Yodh, Science 309, 1207 (2005).
5. J. Daeges, H. Gleiter, J. Perepezko, Phys. Lett. A 119, 79 (1986).

6. J. W. Herman, H. E. Elsayed-Ali, Phys. Rev. Lett. 69, 1228 (1992).

7. F. Banhart, E. Hernández, M. Terrones, Phys. Rev. Lett. 90, 185502 (2003).

8. B. J. Siwick, J. R. Dwyer, R. E. Jordan, R. J. Miller, Science 302, 1382 (2003).

9. H. I. Aaronson, M. Enomoto, ]. K. Lee, Mechanisms of Diffusional Phase Transformations in Metals and Alloys (Taylor \& Francis Group, New York, 2010).

10. Materials and methods are available as supplementary materials on Science Online.

11. V. J. Anderson, H. N. Lekkerkerker, Nature 416, 811 (2002).

12. A. E. Larsen, D. G. Grier, Nature 385, 230 (1997).

13. U. Gasser, E. R. Weeks, A. Schofield, P. N. Pusey, D. A. Weitz, Science 292, 258 (2001).

14. J. R. Savage, D. W. Blair, A. J. Levine, R. A. Guyer, A. D. Dinsmore, Science 314, 795 (2006).

15. Y. Peng, Z.-R. Wang, A. M. Alsayed, A. G. Yodh, Y. Han, Phys. Rev. E 83, 011404 (2011)

16. P. N. Pusey, W. van Megen, Nature 320, 340 (1986).

17. J. C. Crocker, D. G. Grier, J. Colloid Interface Sci. 179, 298 (1996)

18. L. Gómez, A. Dobry, Ch. Geuting, H. T. Diep, L. Burakovsky, Phys. Rev. Lett. 90, 095701 (2003).

19. L. Burakovsky, D. L. Preston, R. R. Silbar, Phys. Rev. B 61, 15011 (2000)

20. M. Forsblom, G. Grimvall, Nat. Mater. 4, 388 (2005)

21. X.-M. Bai, M. Li, Phys. Rev. B 77, 134109 (2008).

22. L. C. Gallington, A. Bongiorno, ]. Chem. Phys. 132 174707 (2010).
23. W. Ostwald, Z. Phys. Chem. 22, 289 (1897).

24. X.-M. Bai, M. Li, Phys. Rev. B 72, 052108 (2005).

25. A. R. Denton, N. W. Ashcroft, Phys. Rev. A 39, 4701 (1989).

26. S. Auer, D. Frenkel, Nature 409, 1020 (2001).

27. R. L. Davidchack, ]. Chem. Phys. 133, 234701 (2010)

28. D. Moroni, P. R. ten Wolde, P. G. Bolhuis, Phys. Rev. Lett. 94, 235703 (2005)

29. H. Zheng et al., Science 324, 1309 (2009)

30. S. Luo et al., Phys. Rev. B 68, 134206 (2003).

31. K. Lu, Y. Li, Phys. Rev. Lett. 80, 4474 (1998).

32. Z. H. Jin, P. Gumbsch, K. Lu, E. Ma, Phys. Rev. Lett. 87, 055703 (2001).

33. ]. Tallon, Nature 342, 658 (1989).

34. H. Fecht, W. Johnson, Nature 334, 50 (1988).

35. Q. Mei, K. Lu, Prog. Mater. Sci. 52, 1175 (2007).

Acknowledgments: This work was supported by the Research Grants Council of Hong Kong (General Research Fund 601208 and 601911), and we thank A. Alsayed and A. Yodh for providing the NIPA colloids.

\section{Supplementary Materials}

www.sciencemag.org/cgi/content/full/338/6103/87/DC1

Materials and Methods

Supplementary Text

Figs. S1 to S4

References

Movies S1 to S6

16 May 2012; accepted 27 August 2012

$10.1126 /$ science. 1224763

\section{A Local Proton Source Enhances $\mathrm{CO}_{2}$ Electroreduction to $\mathrm{CO}$ by a Molecular Fe Catalyst}

\section{Cyrille Costentin, ${ }^{*}$ Samuel Drouet, Marc Robert, Jean-Michel Savéant*}

Electrochemical conversion of carbon dioxide $\left(\mathrm{CO}_{2}\right)$ to carbon monoxide $(\mathrm{CO})$ is a potentially useful step in the desirable transformation of the greenhouse gas to fuels and commodity chemicals. We have found that modification of iron tetraphenylporphyrin through the introduction of phenolic groups in all ortho and ortho' positions of the phenyl groups considerably speeds up catalysis of this reaction by the electrogenerated iron(0) complex. The catalyst, which uses one of the most earth-abundant metals, manifests a CO faradaic yield above $90 \%$ through 50 million turnovers over 4 hours of electrolysis at low overpotential ( 0.465 volt), with no observed degradation. The basis for the enhanced activity appears to be the high local concentration of protons associated with the phenolic hydroxyl substituents.

$\mathrm{T}$ he catalytic reductive transformation of carbon dioxide $\left(\mathrm{CO}_{2}\right)$ to fuels and commodity chemicals is one of the most important contemporary energy and environmental challenges. A highly negative potential is required to inject an electron into $\mathrm{CO}_{2}(1,2)$. Reaction pathways that would go through the intermediacy of the resulting anion radical are therefore quite unreasonable in terms of energy and activation. Focusing on the conversion to carbon monoxide (CO), a number of catalysts - mostly coordina-

Université Paris Diderot, Sorbonne Paris Cité, Laboratoire d'Electrochimie Moléculaire, Unité Mixte de Recherche UniversitéCNRS no. 7591, Bâtiment Lavoisier, 15 Rue Jean de Baïf, 75205 Paris Cedex 13, France.

*To whom correspondence should be addressed. E-mail: cyrille.costentin@univ-paris-diderot.fr (C.C.); saveant@univparis-diderot.fr (].-M.S.) tion complexes of low oxidation state transition metals - have been described (3-5). Among them, iron(0) porphyrins, electrochemically generated from the iron(II) porphyrin by two successive electron uptakes at a mercury or a glassy carbon electrode, are efficient, CO-selective, and durable catalysts provided they are coupled, in the framework of an electron-push-pull process, with Lewis acids (6) or weak Brönsted acids $(7,8)$. On the basis of this observed favorable role of proton donors, we reasoned that acid groups attached to the catalyst molecule should have a strong accelerating effect in view of the large local concentration of acid thus present, which would be impossible to introduce in such amounts in solution in the context of bimolecular reaction.

We have indeed found that modification of tetraphenylporphyrin (TPP) through the introduc- tion of phenolic groups in all ortho and ortho' positions of the TPP phenyl groups, as shown in Fig. 1, leads to a considerable increase of catalytic activity. This is shown in Fig. 2, in which we plot the log of the turnover frequency (turnover number per unit of time), $T O F$, against the overpotential, $\eta$ (difference between the standard potential of the $\mathrm{CO}_{2} / \mathrm{CO}$ couple and the operating electrode potential). The variation of the $T O F$ with the overpotential obtained from cyclic voltammetry of FeTDHPP in $N, N^{\prime}$-dimethylformamide $(\mathrm{DMF})+2 \mathrm{M} \mathrm{H}_{2} \mathrm{O}$ in the presence of a saturating concentration of $\mathrm{CO}_{2}(0.23 \mathrm{M})$ is shown as a thick gray segment.

For such molecular catalytic reactions, in which the catalyst is a well-defined molecule, with a well-defined standard potential, turnover frequency and overpotential were traditionally viewed as independent parameters, not allowing a precise comparison of catalyst performances. The obvious assertion that a good catalyst is characterized by a high $T O F$ and a small $\eta$ (and vice versa) is indeed not very helpful in this purpose. It has recently been shown (9) that turnover frequency and overpotential are in fact linked, for a given catalyst, by a relationship that may be formulated as in Eq. $1(10)(f=F / R T$, where $F$ is Faraday's constant and $R$ the gas constant):

$$
\begin{aligned}
\frac{1}{T O F}= & \frac{1}{k_{\mathrm{cat}}}+\frac{\exp \left[f\left(E-E_{\mathrm{cat}}^{0}\right)\right]}{k_{\mathrm{cat}}}+ \\
& \frac{\sqrt{D}}{k_{\mathrm{S}}} \frac{\exp \left[\frac{f}{2}\left(E-E_{\mathrm{cat}}^{0}\right)\right]}{\sqrt{k_{\mathrm{cat}}}}
\end{aligned}
$$

$k_{\text {cat }}$ is the rate constant of the catalytic reaction, $E_{\text {cat }}^{0}$ the standard potential of the catalyst, $k_{\mathrm{S}}$ its 